\title{
Génie génétique et industrie pharmaceutique
}

\section{Le génie génétique est-il, pour les sociétés pharmaceutiques, un mirage ou un éden? Personne n'ignore plus que l'utilisation des recombinants d'ADN permet aujourd'hui de produire des substances d'intérêt biologique qu'il \\ Une nouvelle forme clinique de l'hystérie : les biotechnologies} serait malaisé ou impossible d'obtenir par les procédés classiques. Cette évidence, cependant, n'est pas une garantie de succès industriel et commercial pour les firmes qui décident de se "lancer» dans l'aventure! Les écueils et les obstacles s'appellent la concurrence étrangère et la " guerre » des brevets, le coût des recherches et du procédé de fabrication, les incertitudes quant aux indications spécifiques des produits obtenus et à l'importance du marché. Trois scientifiques, responsables tous trois de la stratégie industrielle de leur société dans le domaine de la santé et des biotechnologies, nous livrent leurs réflexions et expérience.
There appears to be patent related hysteria building up regarding biotechnology. Il semble se développer une hystérie liée aux brevets en matière de biotechnologie, ainsi débute le très sérieux Drug and Life Sciences Bulletin $\left(n^{\circ} 12\right.$, vol. 3, juillet 1987) édité par la firme d'analyse financière Merril Lynch.

Peut-être ne s'agit-il pas tout à fait des grandes crises décrites par Charcot, néanmoins la fureur procédurière a bel et bien saisi le monde des biotechnologies. Genentech et Hormone Research Foundation sont opposés à propos de la synthèse de l'hormone de croissance, Cetus et Amgen sur l'interleukine 2, Hybritech et Monoclonal Antibodies sur les immuno-essais de type sandwich. Que les services juridiques de Centocor et de Cetus s'agrippent à propos d'une technologie licenciée par un tiers, cela suffit à baisser la cotation des actions du premier, de telle sorte que des investisseurs avisés se précipitent sur cette aubaine. Les brokers* de la Bourse lisent Nature, tandis qur les laboratoires de recherche commencent à déchiffrer le Wall Street Journal.

Le cœur d'un brevet tient en ses «revendications ${ }^{* *}$. Souvent, en matière de biotechnologie, cellesci sont extrêmement vastes et peu étayées. Extrêmement vastes, véri-

\author{
Georges-Anthony Marcel (Roussel Uclaf) \\ Pierre-Étienne Bost (Rhône-Poulenc Santé) \\ Julian E. Davies (Institut Pasteur Production)
}

tables traités de biologie moléculaire, de façon à chercher à couvrir toutes les variantes de l'invention, telles sont les revendications de cette firme, qui se voit rappelée à l'ordre par les examinateurs d'un office des brevets. Certains en disent que leurs revendications en matière d'interféron suffisent à protéger aussi toutes les interleukines! Il en reste toujours quelque chose: l'antériorité non nulle, que des procédures juridiques d'ici quelques années pourront évoquer. Telle autre firme passant du brevet de substance au brevet d'indication, allèguera une efficacité dans l'allergie aux oranges, étayée par deux cas étudiés en ouvert $^{* * *}$ ! Les offices de brevets n'ayant pas encore émis de directives pour juger du caractère suffisant des descriptions en matière d'efficacité clinique, les inventeurs en chambre ont la partie belle. La description, disent les textes juridiques, «doit être suffisante pour permettre à l'homme du métier d'utiliser en pratique les informations données ». Si l'on assimile cette «pratique » à la pratique quotidienne médicale, il faudrait que les informations fournies dans les brevets soient de l'ordre de celles fournies aux autorités d'enregistrement. Cela n'est pas le cas.

Le nombre de molécules naturelles issues des biotechnologies est restreint. D'ores et déjà un très

\footnotetext{
* Broker : courtier.

* Revendications: points spécifiques d'un brevet définissant l'objet de la propriété.

*** En ouvert: dans une étude ni contrôlée, ni comparative.
} 
grand nombre de ces molécules fait l'objet de brevets de substances, et des procédures légales entre firmes ont pu être entreprises. Ces procedures, même lorsqu'elles se soldent par la voie des négociations, entraînent une très grande consommation de temps et d'argent, dont le bénéfice pour la santé publique n'est pas évident. Il n'est pas question de nier que la protection des inventions est primordiale pour la santé publique, qui bénéficie de ces inventions qu'elle doit donc encourager. Mais l'originalité de l'invention ce n'est pas la substance, puisqu'elle est «naturelle», mais le procédé. La brevetabilité même des substances naturelles fait couler beaucoup d'encre. L'accord est loin d'être fait, et certaines ont pu être brevetées (par exemple, la vitamine $B_{12}$ ) et d'autres pas (par exemple, la vitamine $B_{6}$ ).

La conséquence de cette relative paralysie du développement concurrentiel de peptides naturels par crainte des procédures juridiques (patent infringement suits) est double. D'une part faiblesse de l'investissement dans la recherche de nouvelles sources d'ADNc, de nouveaux vecteurs, de nouveaux promoteurs, de nouveaux enhancers, de nouveaux hôtes d'expression, alors que ceux qu'on utilise sont souvent dérivés de tissus cancéreux pour les ADNc et issus de virus nocifs pour les promoteurs ; d'autre part, investissements accrus dans l'élaboration de peptides non naturels, chimères destinées au moins autant à louvoyer entre les récifs des brevets de substance qu'à explorer de nouvelles voies pharmacologiques.

L'Europe des biotechnologies verra d'autant plus sereinement le jour que nous serons en mesure d'affronter ces problèmes de brevets, face à de redoutables concurrents d'Outre-Atlantique dont une des qualités les moins négligeables est de comporter certains cadres possédant une formation supérieure à la fois en biotechno- logie et en droit des brevets. C'est à eux que l'on doit l'apparition, en biotechnologie, de brevets d'utilisation cherchant à bloquer non seulement telle ou telle substance, mais son emploi dans telle ou telle indication.

En conclusion, il faudra simultanément redouter les conséquences d'une trop grande importance donnée aux brevets de substance, propre à provoquer l'essor des chimères issues d'apprentis sorciers, et d'une trop grande légèreté donnée aux brevets d'indication, propre à induire des essais de phase II destinés plus à la couverture d'un nouveau créneau qu'au dose-ranging*

\section{Georges-Anthony Marcel}

Directeur du développement Santé Roussel Uclaf, 35, boulevard des Invalides, 75007 Paris, France et 102 , route de Noisy, 93230 Romainville, France.

\section{Un exemple de stratégie industrielle, Rhône-Poulenc Santé}

En raison du coût important représenté par l'installation d'une équipe de génie génétique complète, peu de sociétés pharmaceutiques françaises peuvent se lancer dans la compétition internationale de cette manière. P.E.Bost, Directeur des Recherches Pharmacie Humaine de Rhône-Poulenc Santé, nous présente ici la stratégie adoptée par son groupe.

Le domaine des biotechnologies recouvre des activités très diverses qui ont en commun la mise en œuvre d'organismes vivants ou de leurs fractions. Récemment, avec l'avènement de la biologie moléculaire et du génie génétique, il est devenu patent que l'innovation pharmaceutique résulterait d'une nouvelle stratégie et entraînerait une réorientation des compétences classiquement utilisées. Ainsi, Rhône-Poulenc Santé (RPS) a pris en compte, dès 1979 , l'apparition du génie génétique en créant une filiale spécialisée.

Plus d'une quinzaine de chercheurs (phD) très souvent titulaires d'une autre formation (médecin, chimiste, agronome, polytechnicien) ont été recrutés. Cette équipe pluridisciplinaire et internationale a pour mission de promouvoir toutes les retombées prévisibles, à court et à long terme, des recombinaisons d'ADN in vitro. De fait, trois groupes complémentaires ont été définis afin de couvrir les domaines des procaryotes et des eucaryotes supérieurs et inférieurs. Bien que leur approche des problèmes techniques reste globalement différente, l'objectif de ces trois groupes est similaire: le clonage de gènes, quelle que soit leur origine, et leur expression dans des systèmes souvent phylogénétiquement éloignés (bactéries, cellules animales ou levures). Le niveau de cette expression est un problème majeur notamment pour l'extrapolation à la production industrielle des résultats obtenus au laboratoire sur l'obtention de protéines d'intérêt médical. Bien qu'historiquement RPS possède un savoir-faire dans ce domaine, il s'est rapidement avéré nécessaire de renforcer les équipes traditionnelles par des chercheurs spécialistes des protéines recombinantes, afin de réaliser le changement d'échelle que nécessite la production industrielle. Ces chercheurs sont extrêmement demandés en raison de leur rareté sur le marché du travail.

Afin de promouvoir les interactions entre ces différentes équipes, un centre de recherche unique va bientôt rassembler tous les chercheurs concernés en un même bâtiment, à Vitry-sur-Seine. RPS attend donc beaucoup du génie génétique pour la production de nouveaux médicaments et pour 
optimiser sa recherche pharmacologique. Par exemple, le clonage des gènes de récepteurs des médiateurs du système nerveux central permet d'envisager de nouvelles approches pour le traitement spécifique de maladies nerveuses: schizophrénie, psychose maniacodépressive, maladie d'Alzheimer, anxiété et insomnie. Dans ce domaine, des progrès considérables vont prochainement voir le jour grâce au développement de nouvelles techniques: mutagenèse dirigée de domaines protéiques, hyper-expression transitoire et conception de médicaments plus spécifiques assistée par ordinateur.

Le clonage des gènes hétérologues n'est pas la seule préoccupation des biotechniciens de RPS. En effet, l'amélioration des souches de micro-organismes qui produisent industriellement antibiotiques et vitamines, est également justiciable des techniques de recombinaisons d'ADN in vitro. Bien que les avancées observées soient moins spectaculaires que celles résultant de l'expression de protéines humaines, elles n'en demeurent pas moins très importantes sur le plan économique.

Par ailleurs, l'Institut Mérieux poursuit la mise au point de vaccins de nouvelle génération : vaccins recombinants, clonés ou non dans le virus de la vaccine; vaccins contre l'herpès, l'hépatite, la rage sylvatique, le SIDA et certaines parasitoses tropicales par le biais de Pasteur-Vaccins.

Historiquement, grâce à la maîtrise simultanée de la chimie et des biotechnologies, la société RPS a toujours pu choisir les stratégies de recherche et de production les plus adaptées au marché pharmaceutique et elle entend continuer demain à jouer un rôle moteur dans ce domaine au niveau mondial

\section{Pierre-Étienne Bost}

Directeur Recherches Pharmacie Humaine, Rhône-Poulenc Santé, 20, avenue Raymond Aron, 92160, Antony, France.

\section{Stratégie industrielle et génie génétique}

$\mathrm{m} / \mathrm{s}$ : les grands groupes pharmaceutiques mis en éveil par l'émergence des techniques liées au génie génétique ont-ils adopté dans ce domaine une stratégie digne de ce nom ou se sont-ils contentés de rester sceptiques face à l'émergence d'une nuée de petites sociétés engagées totalement dans l'exploitation de ces nouvelles techniques?

\begin{tabular}{|c|c|}
\hline \multicolumn{2}{|c|}{$\begin{array}{c}\text { Tableau I } \\
\text { EXEMPLES MONTRANT LE NOMBRE DÉCLARÉ } \\
\text { DE SOCIÉTÉS TRAVAILLANT SUR UNE PROTÉINE DONNÉE }\end{array}$} \\
\hline Interféron gamma & $\begin{array}{l}\text { Amgen, Asahi Chemical, } \\
\text { Biogen, Boehringer Ingelheim, } \\
\text { Daiichi, Seiyaku, Flow, } \\
\text { Genentech, Green Cross, } \\
\text { Immuno Modulators, Interferon } \\
\text { Sciences, Kyowa Hakko, Meiji } \\
\text { Seika, Meloy, Mochida, } \\
\text { Revlon, Roche, Roussel-Uclaf, } \\
\text { Searle, Schering-Plough, } \\
\text { Sclavo, Serono, Shionogi, } \\
\text { Suntory, Takeda, Toray, } \\
\text { Transgène, Yissum }\end{array}$ \\
\hline Interleukine 2 & $\begin{array}{l}\text { Ajinomoto, Amgen, Biogen, } \\
\text { Biotech Research Labs, } \\
\text { Bristol-Myers, Cellular } \\
\text { Products, Cetus, Chiron, Du Pont, } \\
\text { Genetics Institute, } \\
\text { Genex, Immunex, Interferon } \\
\text { Sciences, Interleukin-2 Inc, } \\
\text { Quidel, Roche, Roussel-Uclaf, } \\
\text { Sandoz, Sanofi, Shionogi, } \\
\text { Suntory, Takeda, Transgène, } \\
\text { Yissum, Yoshitomi }\end{array}$ \\
\hline Tissue plasminogen activator & $\begin{array}{l}\text { Baxter-Travenol, Biogen, } \\
\text { Boehringer Ingelheim, } \\
\text { Celltech, Chiron, Ciba-Geigy, } \\
\text { Fujisawa, Genentech, Genetics } \\
\text { Institute, Genex, Green } \\
\text { Cross, Integrated Genetics, } \\
\text { Inveresk, KabiVitrum, Kyowa } \\
\text { Hakko, Mitsubishi, Mitsui } \\
\text { Toatsu, Sankyo, SmithKline, } \\
\text { Toyobo, Yamanouchi }\end{array}$ \\
\hline
\end{tabular}

J.E. Davies: la réponse à cette question est évidemment fonction de l'entreprise. Il y a là, en effet, un problème d'échelle et de structure. Les petites sociétés de génie génétique, telles Genentech, Cetus ou Biogen, sont toutes à la recherche de protéines dont l'intérêt thérapeutique sera démontré le plus rapidement possible. Dans leurs cas, par nécessité, la stratégie se limite au choix du sujet sur lequel vont porter leurs efforts de recherche et aux méthodologies applicables. En effet, si le capital de Biogen peut être estimé à 250 millions de dollars, il faut savoir que des sociétés multinationales comme Schering-Plough ou Merck ont annuellement à 
leur disposition un budget de recherche de 100 et 500 millions de dollars, respectivement. Dans ce contexte, les petites sociétés de génie génétique n'ont pas le droit à l'erreur. Il leur faut identifier une protéine dotée d'un potentiel thérapeutique, s'y accrocher de toutes leurs forces en investissant compétences et capitaux disponibles. Ces sociétés sont donc extrêmement créatives car elles n'hésitent pas à s'engager très tôt sur des produits dont l'intérêt médical est quelquefois spéculatif. Actuellement, si l'on prend ces sociétés dans leur ensemble, leur efficacité est si grande que les protéines découvertes n'ont pas pu encore être toutes testées (Tableau I).

La réalité pharmacologique est parfois douloureuse pour certaines d'entre elles ; d'autant qu'il peut y avoir jusqu'à 20 sociétés travaillant sur le même sujet. Le tumor necrosis factor (TNF) n'a pas d'application en thérapeutique humaine; l'interféron $\alpha$ est indiqué dans la leucémie à tricholeucocytes (autrement dit, un marché très confidentiel) ; l'interféron $\gamma$ a déçu dans le traitement du cancer et de la polyarthrite rhumatoïde; l'interleukine 2 s'avère un bon immunostimulant, mais son indication anticancéreuse revient à 25000 dollars par malade.

La stratégie pour les petites sociétés de génie génétique est alors dramatiquement simple: il faut persévérer, modifier la ou les molécules en question par une mutagenèse plus ou moins dirigée, et espérer jusqu'à la sanction du marché financier. Ceci explique les problèmes de revenus rencontrés actuellement par la plupart des sociétés de ce type. Genentech tire de ses ventes au maximum $10 \%$ de ses revenus. Biogen et Cetus $2 \%$ et Amgen moins de $2 \%$. Cette fragilité financière explique la facilité avec laquelle certaines de ces sociétés ont été rachetées par de grands groupes industriels.

Bien sûr, et heureusement, il y a des succès représentés par l'activateur tissulaire du plasminogène (tPA), le vaccin contre l'hépa- tite B, l'érythropoïétine... et certains de ces produits obtenus par génie génétique sont déjà sur le marché. Une réussite dans ce domaine entraîne beaucoup de convoitise de la part des groupes industriels et des autres petites sociétés de génie génétique travaillant sur le même projet, conduisant respectivement à des prises de participation et à des procès mettant en cause l'invention et l'antériorité de celle-ci. Quelle est la stratégie des « grands de l'industrie pharmaceutique» dans les secteurs dominés par l'application du génie génétique? L'échelle étant différente, il ne s'agit plus de tout miser sur une protéine mais sur un domaine thérapeutique (cardiovasculaire, neurologie, etc.) et donc sur un département constitué de chercheurs spécialistes du domaine. Mais suffit-il d'inonder de capitaux un ou plusieurs départements pour innover et trouver de nouvelles molécules? Globalement, la réponise est non ; $80 \%$ des grands groupes n'ont pas de stratégie de recherche au sens créatif du terme. Il leur manque la flexibilité. En revanche leur puissance financière, leurs énormes moyens de développement, leur permettent de commercialiser efficacement dans le monde entier le produit médicamenteux repéré. Ce jeu du chat et de la souris va conduire quelquesuns de ces grands groupes pharmaceutiques à prendre, très souvent pour une modique somme, le contrôle d'une ou de plusieurs sociétés spécialisées en génie

\section{* Screening : criblage.}

* Les substances naturelles des organismes vivants ont un récepteur plus ou moins spécifique sur lequel elles vont se fixer afin d'induire leur effet. Cette interaction spécifique de type ligand-récepteur peut être déplacée par une molécule synthétique qui va alors inhiber, mimer ou augmenter l'effet induit par le ligand. Cette interaction dans un système donné va permettre d'étiqueter une activité à la molécule synthétique testée et permettre son étude ultérieure dans le traitement d'une maladie donnée. génétique. Le résultat est alors souvent décevant, la rigidité et l'éclatement des structures remplaçant la souplesse et l'autonomie créatrice.

Une stratégie beaucoup mieux adaptée aux groupes industriels consiste à trouver le moyen de mettre en valeur ce qui fait leur richesse, à savoir le stock des molécules synthétisées depuis des années et auxquelles aucune activité pharmacologique n'a pu être reliée. La plupart des firmes pharmaceutiques s'engagent dans cette voie. Afin d'y parvenir, il leur faut définir et mettre au point des tests de screening ${ }^{*}$ beaucoup plus raffinés que ceux utilisés précédemment. Le génie génétique est une des techniques de choix pour se procurer des outils biologiques de type ligand ** (le plus souvent ce sont des hormones), ou récepteur, nécessaires à la mise au point de ces nouveaux tests. Ceux-ci vont alors permettre la découverte de molécules actives dans un stock mondial qui en comporte plusieurs centaines de milliers.

En conclusion, il ne faut pas oublier que le génie génétique peut s'appliquer à bien d'autres domaines que celui de la pharmacie et avoir l'envergure de s'attaquer à des problèmes dont les retombées ne sont pas envisageables à court terme. Ainsi des sociétés comme Monsanto ou d'autres, liées à de grands groupes pétroliers, commencent à s'intéresser à la biomasse. De même, le domaine agro-alimentaire n'a encore été que trop peu investi dans les pays industrialisés en raison des surplus, alors qu'il s'agira probablement d'un problème mondial dans les décennies à venir

\section{Julian E. Davies}

Chef de l'unité de génie microbiologique, Institut Pasteur, département des biotechnologies, 25, rue du Docteur-Roux, 75724 Paris Cedex 15, France. 